\title{
Deciphering the Fine-Tuning of the Retinoic Acid-Inducible Gene-I Pathway in Teleost Fish and Beyond
}

\author{
Raphaël Jami, Emilie Mérour, Annie Lamoureux, Julie Bernard, Jean K. Millet \\ and Stéphane Biacchesi ${ }^{*}$ \\ University Paris-Saclay, INRAE, UVSQ, VIM, Jouy-en-Josas, France
}

OPEN ACCESS

Edited by:

Vincenzo Torraca,

University of London,

United Kingdom

Reviewed by:

Sarah J. Poynter,

University of Waterloo, Canada

Takumi Koshiba,

Fukuoka University, Japan

${ }^{*}$ Correspondence:

Stéphane Biacchesi

stephane.biacchesi@inrae.fr

Specialty section: This article was submitted to Comparative Immunology, a section of the journal

Frontiers in Immunology

Received: 11 March 2021 Accepted: 07 April 2021

Published: 28 April 2021

Citation:

Jami R, Mérour E, Lamoureux A, Bernard J, Millet JK and Biacchesi S (2021) Deciphering the Fine-Tuning of

the Retinoic Acid-Inducible Gene-I Pathway in Teleost Fish and Beyond.

Front. Immunol. 12:679242.

doi: 10.3389/fimmu.2021.679242
Interferons are the first lines of defense against viral pathogen invasion during the early stages of infection. Their synthesis is tightly regulated to prevent excessive immune responses and possible deleterious effects on the host organism itself. The RIG-I-like receptor signaling cascade is one of the major pathways leading to the production of interferons. This pathway amplifies danger signals and mounts an appropriate innate response but also needs to be finely regulated to allow a rapid return to immune homeostasis. Recent advances have characterized different cellular factors involved in the control of the RIG-I pathway. This has been most extensively studied in mammalian species; however, some inconsistencies remain to be resolved. The IFN system is remarkably well conserved in vertebrates and teleost fish possess all functional orthologs of mammalian RIG-I-like receptors as well as most downstream signaling molecules. Orthologs of almost all mammalian regulatory components described to date exist in teleost fish, such as the widely used zebrafish, making fish attractive and powerful models to study in detail the regulation and evolution of the RIG-I pathway.

Keywords: interferon, RIG-I-like receptors (RLRs), innate response, immune homeostasis, fish

\section{INTRODUCTION}

The antiviral innate immune response in vertebrates is mediated by type I interferon (IFN) and its actions as an autocrine signal for the infected cell and as a paracrine "early warning" signal to neighboring cells $(1,2)$. This host response against virus infection is characterized by the induction of a rapid non-specific antiviral state that blocks virus replication and spread. The IFN system is remarkably well conserved in vertebrates which highlights its critical importance (3). Teleost fish possess functional orthologs of pattern-recognition receptors (PRRs). Toll-like receptors (TLRs) and C-type lectin receptors (CLRs) detect pathogens in the extracellular or the endosomal compartments, while retinoic acid-inducible gene-I (RIG-I)-like receptors (RLRs), Nod-like receptors (NLRs), and cytoplasmic DNA sensors serve as intracellular PRRs. These sensors are able to detect distinct viral molecular patterns, such as nucleic acids or viral proteins, collectively known as pathogen-associated molecular patterns (PAMPs). They synergistically trigger the activation of multiple signaling cascades that induce the production of IFN and other cytokines, thereby establishing an antiviral state and shaping an appropriate adaptive immune response. Among the PRRs, RLRs play a key role in sensing viral RNA in the cytosol and are essential in the 
early induction of IFN $(4,5)$. The ability of IFNs to restrict virus replication in mammals is largely mediated through the induction of hundreds of interferon-stimulated genes (ISGs), collectively referred as the "interferome" (6). Similarly, upregulation of ISGs by IFNs in lower vertebrates has been extensively reported. Several studies point to the maintenance of a stable set of core ISGs during evolution (7) and their key functions for fish defense against viruses $(3,8)$.

\section{RIG-I-LIKE RECEPTORS: FROM RNA SENSING TO IFN INDUCTION}

The sensing of non-self-cytosolic RNA is mediated by RLRs which include RIG-I (DDX58) (9), melanoma differentiationassociated gene 5 (MDA5/IFIH1) (10-12), and laboratory of genetics and physiology 2 (LGP2/DHX58) (13, 14). Notably, RIG-I detects viral replication not only in the cytoplasm, but also in the nuclear compartment (15). In mammals, it is now recognized that most if not all viral infections from RNA and DNA viruses can be recognized by RLRs. RIG-I and MDA5 are $\mathrm{DExD} / \mathrm{H}$ box RNA helicases comprising three domains; two $\mathrm{N}$ terminal caspase recruitment domains (CARDs) in tandem involved in signal transduction, a central helicase domain and a C-terminal domain (CTD) critical for RNA recognition and autoinhibition of CARDs (16). LGP2 contains a helicase domain but lacks CARDs and thus a signal-transducing activity. LGP2 is a regulator with distinct effects on RIG-I and MDA5. While LGP2 clearly upregulates the signaling activity of MDA5, its action on RIG-I-mediated antiviral signaling remains unclear $(13,17,18)$. In fact, LGP2 deficiency has different effects depending on the nature of the viral infection $(19,20)$. Nevertheless, LGP2 can associate with the C-terminus of TNF receptor associated factors (TRAFs) and can regulate TRAF activity downstream of RIG-I and MDA5, indicating that LGP2 can suppress both MDA5-dependent and RIG-Idependent signal transduction (21). RLRs are remarkably well conserved in vertebrates and teleost fish possess functional orthologs of human RLRs, including RIG-I, MDA5, and LGP2 $(4,22)$ as well as several downstream molecules (Figure 1A and Table 1). Although identified in many fish species belonging to Cypriniformes (e.g. carp and zebrafish), Siluriformes (e.g. channel catfish) and Salmoniformes (e.g. salmon and trout), RIG-I has not been reported in certain fish of the superclass Acanthopterygii (e.g. medaka, tetraodon, pufferfish, stickleback, sea bream and sea bass). It is still unclear whether the RIG-I gene has been lost in some fish species as it has been reported for chicken (105) and Chinese tree shrew (106).

RIG-I and MDA5 recognize specific RNA features that are not typically found in most cellular RNAs in the cytoplasm of vertebrate cells (107). RIG-I binds preferentially, but not exclusively, to ssRNAs phosphorylated at the 5' end, whereas MDA5 recognizes long dsRNAs. This difference in ligand preference results in specificity for the recognition of distinct virus species. In the resting state, CARDs are sequestered, while upon binding of RNA to CTD and helicase domains, CARDs are released by a conformational change of the molecule. Exposed
CARDs interact with the CARD of the mitochondrial activator of virus signaling (MAVS) protein (IPS-1, VISA or Cardif) (29-32). MAVS is an integral protein of the mitochondrial outer membrane that associates with the mitochondrial membrane via its C-terminal domain and acts as a key determinant of the antiviral signaling cascade. Fish MAVS contains similar domains as those found in mammals, with a $\mathrm{N}$-terminal CARD domain and a C-terminal transmembrane (TM) region, both of which are essential for its antiviral function, as well as a central proline-rich region containing TNF receptor-associated factor (TRAF)binding motifs $(4,25)$. The interaction between RLRs and MAVS induces the recruitment of adaptor proteins, such as TRAF3 or TRAF6, and the activation by phosphorylation of serine/threonine-protein kinases, TANK-binding kinase 1 (TBK1) and inhibitor- $\kappa \mathrm{B}$ kinases. Consequently, IRF3/IRF7 and nuclear factor kappa-light-chain-enhancer of activated $\mathrm{B}$ cells $(\mathrm{NF}-\kappa \mathrm{B})$ transcription factors are activated, translocate from the cytosol to the nucleus and induce the expression of IFNs and inflammatory cytokines.

\section{REGULATION OF RIG-I-LIKE RECEPTORS}

Under homeostatic conditions, IFNs are expressed at very low and often undetectable levels. Given the critical role of the RIG-Imediated IFN induction pathway, a tight regulation is essential to maintain the immune homeostatic balance and to ensure proper termination of the antiviral response in order to avoid extensive tissue damage, chronic inflammation, and autoimmune diseases. Moreover, since most RIG-I pathway components are ISGs and that their overexpression leads to constitutive IFN production, it is clear that cells must regulate them not only at the transcriptional level, but also at post-transcriptional and posttranslational levels. These distinct regulatory mechanisms act on each sensor and downstream molecule to control antiviral signaling. Regulation at the post-transcriptional level includes alternative pre-mRNA splicing leading to functionally distinct proteins (108), long non-coding RNAs (lncRNAs), and micro RNAs (miRNAs) that both serve as important regulators of RLR signal transduction (5). Some lncRNAs have even been shown to directly bind to RLRs. Post-translational modifications (PTMs) involve the covalent linkage of new functional groups to amino acid residues which in turn fine tune protein properties by regulating protein folding, stability, location, and interaction with other molecules. Several regulatory mechanisms mediated by PTMs have been described (109). Among them, phosphorylation and ubiquitination are the best characterized. Other PTMs such as ISGylation (conjugation with the IFNinducible ubiquitin-like protein ISG15), SUMOylation, methylation, acetylation and deamidation have also been reported to control the RIG-I pathway. In addition, several RLR-binding proteins have been identified as important modulators of RLRs for RNA binding (acting as co-receptors), oligomerization, ubiquitination or affecting subcellular localization (5). In addition, spatiotemporal dynamics of MAVS in mitochondria, in mitochondrial-associated endoplasmic reticulum membranes (MAMs), and in 
A

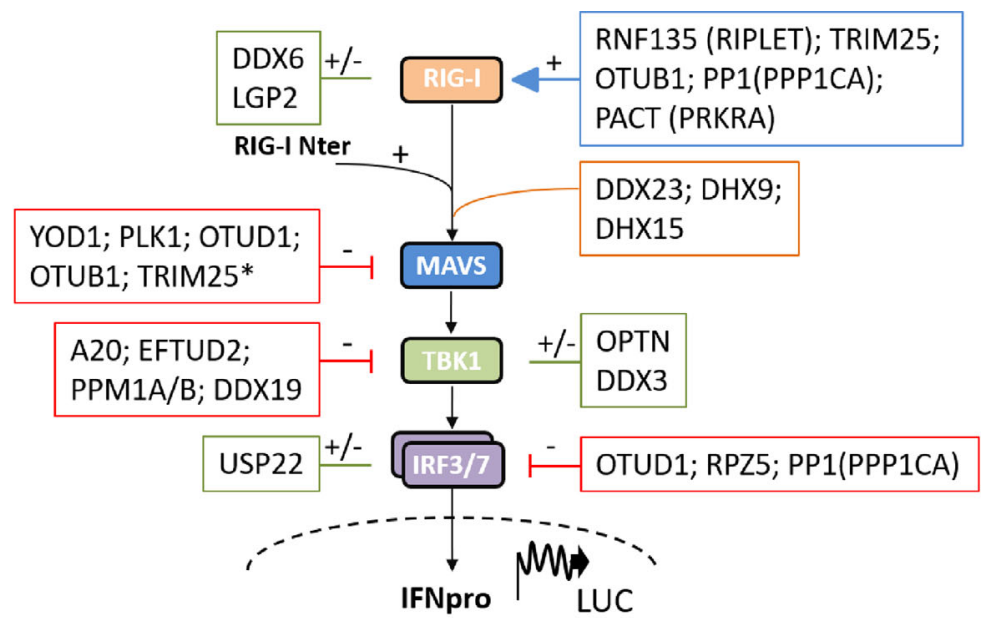

B

C

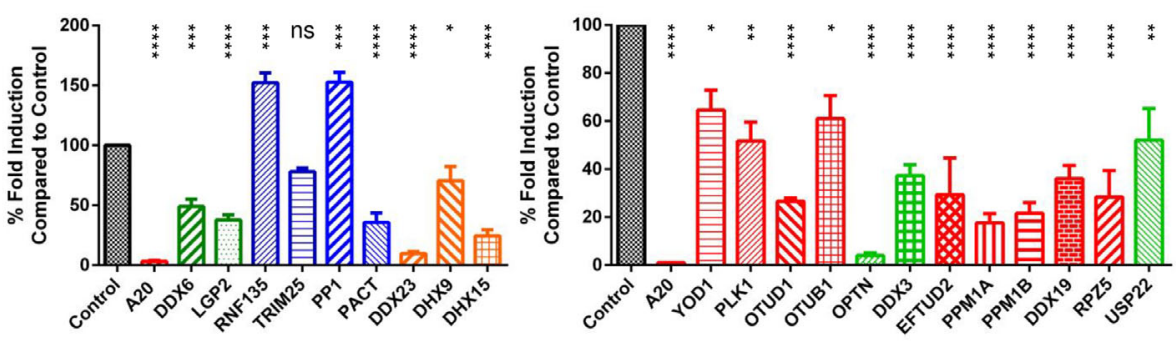

D

E
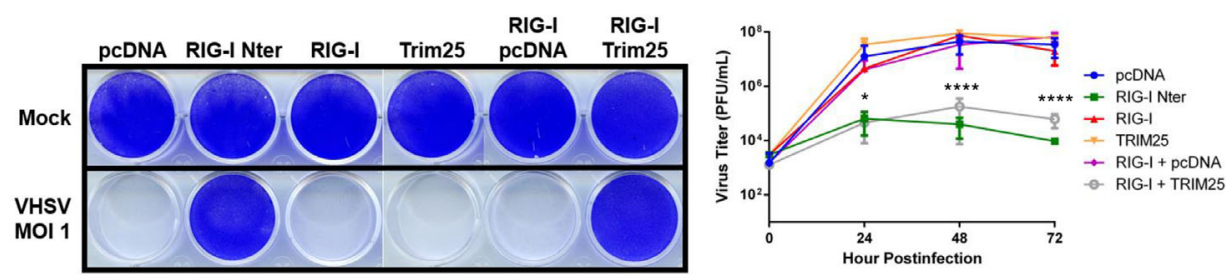

FIGURE 1 | Regulation of RIG-I-mediated signal transduction by a conserved set of cellular proteins in vertebrates. (A) Schematic representation of RIG-I pathway including the main downstream components for signal transduction leading to promoter activation and expression of type-l interferon. A set of cellular regulators that are evolutionarily conserved between fish and mammalian species are placed next to their targets. The effect of each regulator is symbolized by (-) for inhibition (in red), by (+) for activation (in blue), or by (+/-) for ambivalent (in green) reported functions on the RIG-I pathway, based on the literature (mainly from studies with mammalian orthologs; see Table 1). Other cytosolic sensors or co-receptors involved in the RIG-I pathway are boxed in orange. *Although TRIM25 promotes the degradation of MAVS, this step is required for IRF3 phosphorylation by TBK1 (23). (B, C) Fathead minnow orthologs of mammalian regulators were amplified from total RNA extracted from EPC cells, cloned into the eukaryotic expression vectors pcDNA1.1/Amp (Invitrogen) and fully sequenced. Nucleotide sequences of each regulator were deposited in GenBank (see Table 1 for accession numbers). To test their effect on the RIG-I pathway, EPC cells were transfected with the indicated plasmids $(1 \mu \mathrm{g})$ together with a luciferase reporter construct driven by the promoter of IFN1 derived from EPC cells $(1 \mu \mathrm{g})$ and the RIG-I Nter-eGFP inducer and internal transfection control construct $0.5 \mu \mathrm{g}$ in (B) or $1 \mu \mathrm{g}$ in (C), as previously described (24). Twenty-four hours after transfection, the cells were lysed for luciferase assays. Luciferase activity was measured and normalized to eGFP fluorescence. No significant variation in eGFP expression was observed between each condition. The percentage of fold-induction were calculated as the ratio of stimulated (+ RIG-I Nter) versus unstimulated (- RIG-I Nter) conditions and compared to the induction control (RIG-I Nter + empty vector). Means of at least three independent experiments are shown together with the standard errors. The color coding used for the histograms is the same as the one used in panel (A) For statistical analysis, a comparison between groups was performed with a one-way ANOVA and Tukey's multiple comparison tests using GraphPad Prism (GraphPad, San Diego, CA). Groups that are not significantly different from each other are denoted ns $(P>0.05)$, whereas those that are significantly different are denoted ${ }^{*}(P<0.05)$, ${ }^{* *}(P<0.01)$, ${ }^{* \star}(P<0.001)$ or ${ }^{\star \star * \star}(P<0.0001)$. (D, E) EPC cells were transfected with the indicated plasmids ( $2 \mu \mathrm{g}$ each) or an empty vector (pcDNA1.1/Amp) as a control, as previously described (25). All transfection mixtures were adjusted with an empty vector to contain an equal amount of plasmid DNA. Twenty-four hours after transfection, cells were infected with a fish novirhabdovirus, viral hemorrhagic septicemia virus (NHSV) at an MOI of 1 and incubated at $15^{\circ} \mathrm{C}$. Cell monolayers were stained with crystal violet 3 days postinfection (D). The culture supernatants from infected cells were collected at different times postinfection and the viral titer was determined by plaque assay (E). Each time point is represented by two independent experiments, and each virus titration was performed in duplicate. Average values are shown. The standard errors were calculated and the error bars are shown. Asterisks indicate significant difference $\left({ }^{*} \mathrm{p}<0.05 ;{ }^{* \star \star} \mathrm{p}<0.0001\right)$ as determined by two-way ANOVA and Tukey's multiple comparison tests. ns, not significant. 
TABLE 1 | Pimephales promelas RIG-I pathway components and orthologs.

RIG-I Pathway Components of Pimephales promelas

\begin{tabular}{|c|c|c|c|c|c|c|}
\hline Full Name & $\begin{array}{c}\text { Symbol } \\
\text { (Synonym) }\end{array}$ & $\begin{array}{l}\text { Target/ } \\
\text { Partner }\end{array}$ & GenBank \# & $\begin{array}{l}\text { Homo sapiens } \\
\text { GenelD }\end{array}$ & $\begin{array}{l}\text { Danio rerio } \\
\text { GenelD }\end{array}$ & $\begin{array}{c}\text { Selected } \\
\text { References }\end{array}$ \\
\hline DExD/H-box helicase 58 & DDX58 (RIG-I) & MAVS & FN394062 & 23586 & 100333797 & $(9,25,26)$ \\
\hline DDX58 CARD domains & RIG-I Nter & MAVS & FN178456 & & & \\
\hline Interferon induced with helicase C domain 1 & $\mathrm{IFIH1}(\mathrm{MDA} 5)$ & MAVS & MG799354 & 64135 & 565759 & $(10-12,27,28)$ \\
\hline Mitochondrial antiviral signaling protein & $\begin{array}{l}\text { MAVS (IPS1, CARDIF, } \\
\text { VISA) }\end{array}$ & $\begin{array}{l}\text { TRAF3 } \\
\text { TBK1 }\end{array}$ & FN178455 & 57506 & 562867 & $(25,29-33)$ \\
\hline TANK binding kinase 1 & TBK1 & IRF3/7 & LT174673 & 29110 & 692289 & $(24,34-38)$ \\
\hline Interferon regulatory factor 3 & IRF3 & $\begin{array}{l}\text { IFN } \\
\text { promoter }\end{array}$ & MN781134 & 3661 & 564854 & $(39-44)$ \\
\hline Interferon regulatory factor 7 & IRF7 & $\begin{array}{l}\text { IFN } \\
\text { promoter }\end{array}$ & MN781135 & 3665 & 393650 & $(34,45-47)$ \\
\hline Interferon 1 promoter region & IFN1 promoter & - & HE856618 & IFN $\alpha / \beta$ promoter & DQ855952 & $(48-50)$ \\
\hline Interferon 1 & IFN1 & $\begin{array}{l}\text { IFN } \\
\text { receptor }\end{array}$ & FN178457 & $\mathrm{IFN} \alpha / \beta$ & 360134 & $(2,25,51,52)$ \\
\hline \multicolumn{7}{|c|}{ RIG-I Pathway Regulators/Co-receptors/Receptors of Pimephales promelas } \\
\hline DExH-box helicase 58 & DHX58 (LGP2) & RIG-I & MW591879 & 79132 & 100148871 & $\begin{array}{c}(13,17,19,21,27,49, \\
53-55)\end{array}$ \\
\hline DEAD-box helicase 6 & DDX6 & RIG-I & MW591868 & 1656 & 564633 & $(56,57)$ \\
\hline Ring finger protein 135 & RNF135 (RIPLET) & RIG-I & MW591864 & 84282 & 101882927 & $(58-63)$ \\
\hline Tripartite motif containing 25 & TRIM25 & RIG-I & LT174676 & 7706 & 393144 & $(23,64-66)$ \\
\hline $\begin{array}{l}\text { OTU deubiquitinase, ubiquitin aldehyde } \\
\text { binding } 1\end{array}$ & OTUB1 & $\begin{array}{l}\text { RIG-I } \\
\text { TRAF3 }\end{array}$ & MW591878 & 55611 & 436684 & $(67-69)$ \\
\hline $\begin{array}{l}\text { Protein phosphatase } 1 \text { catalytic subunit } \\
\text { alpha }\end{array}$ & PPP1CA (PP1A) & $\begin{array}{l}\text { RIG-I } \\
\text { IRF3/7 }\end{array}$ & MW591866 & 5499 & 407980 & $(70,71)$ \\
\hline $\begin{array}{l}\text { Protein activator of interferon induced } \\
\text { protein kinase } A\end{array}$ & PRKRA (PACT) & RIG-I & MW591865 & 8575 & 557370 & $(18,72)$ \\
\hline DEAD-box helicase 23 & DDX23 & MAVS & MW591869 & 9416 & 334283 & (73) \\
\hline DExH-box helicase 9 & $\mathrm{DHX9}$ & MAVS & MW591876 & 1660 & 568043 & $(74-76)$ \\
\hline DEAH-box helicase 15 & DHX15 & MAVS & MW591867 & 1665 & 321931 & $(77-79)$ \\
\hline YOD1 deubiquitinase & YOD1 (OTUD2) & MAVS & MW591873 & 55432 & 550411 & $(80)$ \\
\hline Polo like kinase 1 & PLK1 & MAVS & MW591874² & 5347 & 280649 & $(81)$ \\
\hline OTU deubiquitinase 1 & OTUD1 & $\begin{array}{l}\text { MAVS } \\
\text { IRF3 }\end{array}$ & MW591870 & 220213 & 100537398 & $(82,83)$ \\
\hline DEAD-box helicase 3 & DDX3 & $\begin{array}{l}\text { MAVS } \\
\text { TBK1 }\end{array}$ & LT174679 & 1654 & 566947 & $(24,75,84-88)$ \\
\hline TNF alpha induced protein 3 & TNFAIP3 (A20) & TBK1 & LT984694 & 7128 & 564497 & $(89-91)$ \\
\hline $\begin{array}{l}\text { Elongation factor Tu GTP binding domain } \\
\text { containing } 2\end{array}$ & EFTUD2 & TBK1 & LT174678 & 9343 & 393480 & $(24,92)$ \\
\hline $\begin{array}{l}\text { Protein phosphatase, Mg2+/Mn2+ } \\
\text { dependent } 1 \mathrm{~A}\end{array}$ & PPM1A & TBK1 & LT174675 & 5494 & 30704 & $(24,93-95)$ \\
\hline $\begin{array}{l}\text { Protein phosphatase, Mg2+/Mn2+ } \\
\text { dependent } 1 \mathrm{~B}\end{array}$ & PPM1B & TBK1 & LT174674 & 5495 & 100003481 & \\
\hline DEAD-box helicase 19 & DDX19 & TBK1 & MW591875 & 55308 & 192339 & (96) \\
\hline Optineurin & OPTN & TBK1 & MW591877 & 10133 & 336159 & $(97-101)$ \\
\hline Rapunzel 5 & $\mathrm{RPZ5}$ & IRF7 & MW591871 & No ortholog & 100003142 & $(24,102)$ \\
\hline Ubiquitin specific peptidase 22 & USP22 & IRF3 & MW591872 & 23326 & 692275 & $(103,104)$ \\
\hline
\end{tabular}

${ }^{1}$ GenBank accession number; ${ }^{2}$ from Danio rerio; new sequences deposited in the GenBank database are in bold.

peroxisomes regulates RLR-mediated signaling (110). Therefore, the integrity of these subcellular compartments together with their own regulation indirectly act on $\operatorname{RLR}$ function $(111,112)$. A few examples of such mechanisms have been described in fish cells, mainly miRNA-mediated regulation and alternative splicing isoforms of RLR components $(113,114)$.

In order to explore the degree of conservation of these regulatory mechanisms among vertebrates, we cloned and fully sequenced 22 genes of fathead minnow (Pimephales promelas) encoding orthologs of human proteins described as important regulators of the RIG-I pathway (Table 1). Fathead minnow is a relevant fish species for at least two reasons: 1) EPC cells
(Epithelioma Papulosum Cyprini; ATCC CRL-2872), the most widely used fish cell line in virology, is derived from this fish species (115), and 2) fathead minnow belongs to the family Cyprinidae together with the zebrafish (Danio rerio), an animal model offering great potential for the study of human and fish viral diseases and the development of antiviral drugs (116-118). This list of 22 fish orthologs is far from exhaustive and only represents a small fraction of proteins described as modulating RIG-I-mediated IFN expression $(109,119)$. Nevertheless, these orthologs are of importance because they correspond to human proteins acting on the RLR pathway via three key modes of action: phosphorylation, ubiquitination, and RLR-binding. 


\section{REGULATION BY KINASES AND PHOSPHATASES}

Phosphorylation is a reversible PTM of proteins in which serine, threonine or tyrosine residues are modified by a kinase by the addition of a covalently bonded phosphate group (109). Phosphorylation results in a structural conformation change of a protein, often modifying its function to become activated or deactivated. The reverse reaction of phosphorylation is called dephosphorylation, and is catalyzed by phosphatases. Phosphorylation regulates almost all components of the RLR pathway. In resting cells, RIG-I is negatively regulated by phosphorylation by several kinases keeping RIG-I in a nonactivated state. When viral RNAs are detected, the CARDs of RIG-I are rapidly dephosphorylated by protein phosphatase 1 (PP1A), thus activating the sensor (70). However, PP1A is also able to dephosphorylate IRF3/7 leading to an inhibition of RIGI-mediated signal transduction at a downstream level (71). Fish PP1A is highly conserved and share $90 \%$ sequence identity at the amino acid (aa) level with its human ortholog. To determine fish PP1A action on RIG-I-mediated IFN expression, we tested its ectopic overexpression in a cell-based luciferase reporter system (Figure 1B). As previously published (48), the expression of a constitutively active form of RIG-I (RIG-I Nter; in which the C-terminal repressor domain maintaining the protein in an inactive state is deleted) significantly activates the IFN1 promoter of EPC cells. As a control, the co-expression RIG-I Nter with A20, a negative feedback regulator of the RLR signaling (89), drastically reduced the induction. In contrast, co-expression of PP1A with RIG-I Nter significantly increase IFN1 promoter activation, indicating that fish PP1A share a common function with its mammalian orthologs by enhancing RIG-I activity. MAVS activation is also regulated by phosphorylation (120). Polo-like kinase 1 (PLK1) has been reported to negatively regulate MAVS (81). PLK1 does not directly phosphorylate MAVS but, rather, requires phosphorylation of MAVS for docking and disrupting the MAVS-TRAF3 interaction. Fish PLK1 is well conserved (71\% aa sequence identity) and also exerts a negative regulatory role on MAVS (Figure 1C). The last example is TBK1. As a critical kinase involved in IFN expression, the activity of TBK1 must be tightly regulated. Because TBK1 activation occurs by trans-autophosphorylation, phosphatases play a critical role in the control of TBK1 activity. Two Ser/Thr protein phosphatases, PPM1A and PPM1B, have been reported to target TBK1 and MAVS for dephosphorylation and to down regulate signaling mediated by cytosolic nucleotide sensing in fish and mammalian species (Figure 1C) (24, 93-95).

\section{REGULATION BY UBIQUITIN LIGASES AND DEUBIQUITINASES}

Ubiquitination is the covalent and reversible addition of ubiquitin to lysine residues on a protein substrate (121).
Ubiquitin is itself an $8.5 \mathrm{kDa}$ protein composed of 76 amino acids. Ubiquitination is catalyzed by three distinct classes of enzymes: ubiquitin-activating enzymes (E1), ubiquitinconjugating enzymes (E2) and ubiquitin ligases (E3), on which lies most of the substrate specificity. Lysine residues can be modified with a single ubiquitin (monoubiquitination) or chains of ubiquitin (polyubiquitination). Different types of ubiquitin chains are thus generated based on the seven lysine residues present on ubiquitin. Among them, K48-linked ubiquitin chains target protein for proteasome degradation while K63-linked ubiquitin chains mediate protein-protein interactions. Ubiquitination is a reversible and dynamic event, since the conjugated ubiquitin chains can be cleaved by a family of ubiquitin-specific proteases, termed deubiquitinases (DUBs). More than 600 and 100 genes encoding putative E3 ligases and DUBs, respectively, have been annotated in the human genome, indicating the ubiquitous importance and specificity of these PTMs in the control of cellular processes. In the RLR pathway, most of the sensors, adaptor proteins, and kinases are ubiquitinated to efficiently activate or repress IFN production.

RIG-I is finely regulated by ubiquitination which is critical for its activation and degradation. Tripartite motif containing 25 (TRIM25) was the first identified enzyme to catalyze the conjugation of K63-linked ubiquitin chains to RIG-I CARDs (64, 65). Ring finger protein 135 (RNF135/RIPLET), another ubiquitin ligase, is also involved in K63-linked polyubiquitination at multiple sites in CARDs and CTD leading to the activation of RIG-I (58-60). Whether RNF135 promotes TRIM25 binding on RIG-I in a sequential ubiquitination process or RNF135 by itself, without involvement of TRIM25, is essential for RIG-I activation is still unclear $(61,62)$. However, TRIM25 is also capable of promoting K48-linked ubiquitination and degradation of MAVS. The proteasomal degradation of MAVS is required to release the signaling complex into the cytosol, allowing IRF3 phosphorylation by TBK1 (23). Zebrafish orthologs of TRIM25 and RIPLET have also been reported as positive regulators of RIG-I $(63,66)$. Figure 1B shows that fish RNF135 has an enhancing effect on the activity of RIG-I CARDs, whereas TRIM25 has no effect. Nevertheless, TRIM25 co-expression with full-length RIG-I is required to protect EPC cells against a viral infection and to inhibit viral production (Figures 1D, E), highlighting that fish RNF135 and TRIM25 are both positive regulators of the RLR pathway.

Several DUBs of ovarian tumor proteases (OTUs) and ubiquitin-specific proteases (USPs) families, have been described as important regulators of RLR pathway. Among them, mammalian and fish A20 has been shown to be a strong inhibitor of the RLR signaling (Figures 1C, D) (89-91). In addition, the function of OTUB1, OTUD1, YOD1, and USP22 fish orthologs was investigated (Table 1). They all have significant inhibitory effects on signal transduction by RIG-I CARDs (Figure 1C). Fish OTUD1 has the strongest effect, likely by mediating the targeted degradation of the MAVS/TRAF3/ TRAF6 signalosome as well as by reducing the DNA binding capacity of IRF3, as described in mammals $(82,83)$. YOD1, which 
acts at a later step along the pathway to abrogate the formation of prion-like aggregates of MAVS (80), has a limited effect on IFN promotor induction at an early time point post-stimulation. In contrast, mammalian OTUB1 and USP22 were reported with opposite regulatory effects on the RLR pathway $(67-69,103,104)$. The inhibitory effect observed after ectopic expression of the fish orthologs may be a result of the inherent bias associated with the overexpression of enzymatically-active protein, mislocalization and inadequate cell type and does not allow to distinguish the opposite functions previously described in mammals.

\section{REGULATION BY RLR-BINDING PROTEINS}

The RLR pathway is regulated by multiple host factors. Protein activators of PKR (PACT, also known as protein activator of interferon induced protein kinase A) binds to RIG-I CTD and enhances RIG-I signaling in part by stimulating RIG-I ATPase and helicase activities (72). Moreover, recent studies have indicated that the PACT-LGP2 interaction was necessary to regulate the responses mediated by RIG-I and MDA5 (18, 122). As for mammals, the role of fish LGP2 in RLR signaling is unclear. It appears that depending on the nature of the splicing isoform, LGP2 can have a negative or a positive effect on the RIG-I pathway $(27,49,53-55)$. The $d h x 58$ cDNA amplified from EPC cells encodes LGP2 protein which exerts a strong inhibition on signaling mediated by RIG-I CARDs (Figure 1B). Moreover, a similar inhibition is observed during expression of RIG-I CARDs together with PACT (Figure 1B). This is in contrast with PACT's enhancing function observed in mammals. However, fish PACT only shares $44 \%$ aa sequence identity with human PACT. Another dsRNA-binding protein, TARRNA-binding protein (TRBP), which shares 39\% protein sequence identity with PACT with a similar structure, has recently been reported as an inhibitor of RIG-I signaling (123). Because fish PACT still retains some degree of relatedness to both human proteins, PACT and TRBP, it cannot be excluded that PACT acts as a negative regulator of RIG-I in fish.

The involvement of multiple RNA helicases in RLR signaling has been demonstrated, as recently reviewed by Taschuk and Cherry (124). For instance, DDX6, DHX9, DDX3, and DHX15 can function as co-sensors of RIG-I or as RLR-independent sensors of nucleic acids through interaction with MAVS (56, 74, 77-79, 84-86). DHX9, DHX15, and DDX23 have been recently described as cytoplasmic viral RNA sensors in the lancelet (amphioxus) (73). However, limitations or contradictions have been reported concerning their role in IFN and ISGs production. DDX6 is also described as a suppressor of ISGs (57). DHX9 is an important viral dsRNA sensor only in myeloid dendritic cells (74). DHX15 contributes to the activation of NF- $\kappa$ B but not IRF3 in response to RNA virus infection (78). DDX3, for which multiple roles as a pro- or antiviral factor were identified (84), has recently been described as an inhibitor of IFN production during arenavirus infection (87). Fish orthologs are highly conserved and share at least $69 \%$ aa sequence identity with human proteins. Fish DDX3 and DHX9 bind dsRNA (75) and DHX9 is a potential sensor for DNA virus infection in vivo (76). Fish DDX3 is a binding partner for the nonvirion (NV) proteins of two fish novirhabdoviruses, suggesting that DDX3 plays an important role in either enhancing innate immunity or promoting virus replication (24). Moreover, the overexpression of fish DDX3 alone seems to induce the IFN promoter (88). In our cell-based reporter system, a negative effect on RIG-I CARDs-mediated signaling was observed for DDX6, DHX9, DDX3, DHX15, and DDX23 (Figures 1B, C), probably through a competition for MAVS adaptor or another mechanism yet to be further investigated. In any case, these RNA helicases are potentially involved in the innate immune system of vertebrates. Finally, another RNA helicase, DDX19, has been shown as a negative regulator of IFN production (96). Mechanistically, DDX19 does not sense viral RNA but inhibits the phosphorylation of IRF3 by TBK1. DDX19 is highly conserved between fish and mammals (sharing $86 \%$ aa sequence identity) and share the same inhibitory effect on the RLR pathway (Figure 1C).

The optineurin (OPTN) is another regulator of the RLR pathway but its action is controversial. Although OPTN was initially reported to negatively regulate IFN induction (97), other studies indicated that OPTN was necessary for optimal TBK1 and IRF3 activation $(98,101)$. However, recent studies pointed out a crucial role for OPTN in dampening the IFN response (99, 125). Moreover, chicken OPTN has been reported as an inhibitor of MDA5-mediated IFN production (100). As shown in the Figure 1C, fish OPTN has also an important inhibitory effect on RIG-I-mediated induction of the IFN promoter.

The function of the NV proteins of two novirhabdoviruses in the inhibition of the host immune response has been described using an interactome proteomics approach (24). Among the cellular partners of NV, PPM1B was shown to be specifically recruited to terminate RIG-I-mediated IFN induction. In addition to DDX3, two other proteins were identified to be likely involved in the RLR pathway: the elongation factor $\mathrm{Tu}$ GTP binding domain containing 2 (EFTUD2) and the rapunzel 5 protein (RPZ5). EFTUD2 was discovered to restrict infection by hepatitis $\mathrm{C}$ virus (HCV) through IFN-independent stimulation of the innate immune response (92). EFTUD2 upregulates RIG-I expression by pre-mRNA splicing. Fish EFTUD2 is highly conserved with its mammalian counterpart (89\% aa sequence identity) but its overexpression does not protect fish cells against rhabdovirus infection in contrast to its human ortholog that protects human cells against HCV (data not shown). Surprisingly, overexpression of EFTUD2 has a negative effect on RIG-I-mediated IFN expression in fish cells (Figure 1C), a finding that requires further investigation. Unlike most of the factors described above, RPZ5 has no mammalian or bird orthologs. Zebrafish RPZ5 has recently been implicated in blocking RLR-mediated IFN induction by mediating the degradation of phosphorylated IRF7 (102). In Figure 1C, we confirm the inhibitory effect of fish RPZ5 on the RIG-I pathway and its uniqueness among teleost fish. 


\section{CONCLUSIONS AND PERSPECTIVES}

The IFN system is remarkably well conserved in vertebrates and it is remarkable that teleost fish possess most post-transcriptional and post-translational regulatory mechanisms of the RLR signaling pathway as described in mammals. Thus, these multilevel regulatory mechanisms were selected very early on and maintained throughout the evolution of vertebrates indicating their crucial role in the control of immune homeostasis for these organisms. Although numerous regulators have been reported in mammals, underlying the complexity and the relative redundancy of these mechanisms, their distinctive roles and functional differences depending on the cell type considered (e.g. immune versus epithelial cells), their own regulation, and their sequential chronology required to orchestrate the RLR signaling remain elusive, and in some cases, opposite functions have been reported for a same effector. In teleost fish, characterization of the components of the RLR pathway and factors involved in its fine tuning has begun but the overall picture is still poorly understood and is mainly modeled on the knowledge acquired from studies based on mammalian systems. The experimental approaches to study the innate immune system in fish has long been based on the overexpression in cell lines of identified genes with the known benefits and limitations of a such screening method. However, with the adaptation of the CRISPR/Cas9 genome editing for fish cells $(126,127)$, gene knock out studies will be greatly improved compared to the low efficiency and biases observed with RNA silencing (128). Moreover, the in vivo relevance of these factors in antiviral immunity still needs to be addressed since their description was exclusively done in vitro in non-immune cells. Since many decades, zebrafish is an important animal model in biomedical research due to multiple advantages including low maintenance cost, high fecundity, short generation time, small size, optical transparency of embryos, and a relatively high degree of conservation with human genes (Table 1) $(129,130)$. Together with the large available collection of transgenic lines and the relative ease to silence or overexpress specific genes, these

\section{REFERENCES}

1. Dahle MK, Jorgensen JB. Antiviral Defense in Salmonids - Mission Made Possible? Fish Shellfish Immunol (2019) 87:421-37. doi: 10.1016/ j.fsi.2019.01.043

2. Fensterl V, Sen GC. Interferons and Viral Infections. Biofactors (2009) 35 (1):14-20. doi: 10.1002/biof.6

3. Langevin C, Aleksejeva E, Passoni G, Palha N, Levraud JP, Boudinot P. The Antiviral Innate Immune Response in Fish: Evolution and Conservation of the IFN System. J Mol Biol (2013) 425(24):4904-20. doi: 10.1016/ j.jmb.2013.09.033

4. Chen SN, Zou PF, Nie P. Retinoic Acid-Inducible Gene I (Rig-I)-Like Receptors (Rlrs) in Fish: Current Knowledge and Future Perspectives. Immunology (2017) 151(1):16-25. doi: 10.1111/imm.12714

5. Rehwinkel J, Gack MU. Rig-I-Like Receptors: Their Regulation and Roles in RNA Sensing. Nat Rev Immunol (2020) 20(9):537-51. doi: 10.1038/s41577020-0288-3

6. Shaw AE, Hughes J, Gu Q, Behdenna A, Singer JB, Dennis T, et al. Fundamental Properties of the Mammalian Innate Immune System advantages make zebrafish a model of choice for studying the spatio-temporal regulatory mechanisms of the RLR pathway. An improved understanding of the precise mechanisms of regulation in different viral and animal species and cell types will enable the development of novel therapeutic strategies against infectious diseases, immunological disorders, and cancer.

\section{DATA AVAILABILITY STATEMENT}

The datasets presented in this study can be found in online repositories. The names of the repository/repositories and accession number(s) can be found below: https://www.ncbi. nlm.nih.gov/genbank/, MN781134 MN781135 MW591879 MW591868 MW591864 MW591878 MW591866 MW591865 MW591869 MW591876 MW591867 MW591873 MW591874 MW591870 MW591875 MW591877 MW591871 MW591872.

\section{AUTHOR CONTRIBUTIONS}

All authors listed have made a substantial, direct and intellectual contribution to the work, and approved it for publication.

\section{FUNDING}

This work was supported by the institut de recherche pour l'agriculture, l'alimentation et l'environnement (INRAE). RJ is the recipient of a Ph.D. fellowship from the Doctoral School ABIES, AgroParisTech.

\section{ACKNOWLEDGMENTS}

We thank INRAE for its financial support. We thank Sandra Souto and Alain Le Coupanec for helpful discussions.

Revealed by Multispecies Comparison of Type I Interferon Responses. PloS Biol (2017) 15(12):e2004086. doi: 10.1371/journal.pbio.2004086

7. Levraud JP, Jouneau L, Briolat V, Laghi V, Boudinot P. Ifn-Stimulated Genes in Zebrafish and Humans Define an Ancient Arsenal of Antiviral Immunity. J Immunol (2019) 203(12):3361-73. doi: 10.4049/jimmunol. 1900804

8. Robertsen B. The Role of Type I Interferons in Innate and Adaptive Immunity Against Viruses in Atlantic Salmon. Dev Comp Immunol (2018) 80:41-52. doi: 10.1016/j.dci.2017.02.005

9. Yoneyama M, Kikuchi M, Natsukawa T, Shinobu N, Imaizumi T, Miyagishi $\mathrm{M}$, et al. The RNA Helicase RIG-I has an Essential Function in DoubleStranded RNA-Induced Innate Antiviral Responses. Nat Immunol (2004) 5 (7):730-7. doi: 10.1038/ni1087

10. Gitlin L, Barchet W, Gilfillan S, Cella M, Beutler B, Flavell RA, et al. Essential Role of Mda-5 in Type I Ifn Responses to Polyriboinosinic:Polyribocytidylic Acid and Encephalomyocarditis Picornavirus. Proc Natl Acad Sci USA (2006) 103(22):8459-64. doi: 10.1073/pnas.0603082103

11. Kang DC, Gopalkrishnan RV, Wu Q, Jankowsky E, Pyle AM, Fisher PB. Mda-5: An Interferon-Inducible Putative Rna Helicase With DoubleStranded RNA-Dependent Atpase Activity and Melanoma Growth- 
Suppressive Properties. Proc Natl Acad Sci USA (2002) 99(2):637-42. doi: $10.1073 /$ pnas. 022637199

12. Kato H, Takeuchi O, Sato S, Yoneyama M, Yamamoto M, Matsui K, et al. Differential Roles of MDA5 and RIG-I Helicases in the Recognition of RNA Viruses. Nature (2006) 441(7089):101-5. doi: 10.1038/nature04734

13. Rothenfusser S, Goutagny N, DiPerna G, Gong M, Monks BG, Schoenemeyer A, et al. The RNA Helicase Lgp2 Inhibits TLR-independent Sensing of Viral Replication by Retinoic Acid-Inducible Gene-I. J Immunol (2005) 175(8):5260-8. doi: 10.4049/jimmunol.175.8.5260

14. Yoneyama M, Kikuchi M, Matsumoto K, Imaizumi T, Miyagishi M, Taira K, et al. Shared and Unique Functions of the DExD/H-box Helicases RIG-I, MDA5, and LGP2 in Antiviral Innate Immunity. J Immunol (2005) 175 (5):2851-8. doi: 10.4049/jimmunol.175.5.2851

15. Liu G, Lu Y, Thulasi Raman SN, Xu F, Wu Q, Li Z, et al. Nuclear-Resident RIG-I Senses Viral Replication Inducing Antiviral Immunity. Nat Commun (2018) 9(1):3199. doi: 10.1038/s41467-018-05745-w

16. Kowalinski E, Lunardi T, McCarthy AA, Louber J, Brunel J, Grigorov B, et al. Structural Basis for the Activation of Innate Immune Pattern-Recognition Receptor RIG-I by Viral Rna. Cell (2011) 147(2):423-35. doi: 10.1016/ j.cell.2011.09.039

17. Rodriguez KR, Bruns AM, Horvath CM. MDA5 and LGP2: Accomplices and Antagonists of Antiviral Signal Transduction. J Virol (2014) 88(15):8194200. doi: 10.1128/JVI.00640-14

18. Sanchez David RY, Combredet C, Najburg V, Millot GA, Beauclair G, Schwikowski B, et al. Lgp2 Binds to PACT to Regulate Rig-I- and MDA5mediated Antiviral Responses. Sci Signal (2019) 12(601). doi: 10.1126/ scisignal.aar3993

19. Satoh T, Kato H, Kumagai Y, Yoneyama M, Sato S, Matsushita K, et al. LGP2 is a Positive Regulator of RIG-I- and MDA5-mediated Antiviral Responses. Proc Natl Acad Sci USA (2010) 107(4):1512-7. doi: 10.1073/pnas.0912986107

20. Venkataraman T, Valdes M, Elsby R, Kakuta S, Caceres G, Saijo S, et al. Loss of DExD/H Box RNA Helicase LGP2 Manifests Disparate Antiviral Responses. J Immunol (2007) 178(10):6444-55. doi: 10.4049/ jimmunol.178.10.6444

21. Parisien JP, Lenoir JJ, Mandhana R, Rodriguez KR, Qian K, Bruns AM, et al. Rna Sensor Lgp2 Inhibits Traf Ubiquitin Ligase to Negatively Regulate Innate Immune Signaling. EMBO Rep (2018) 19(6). doi: 10.15252/ embr.201745176

22. Mojzesz M, Rakus K, Chadzinska M, Nakagami K, Biswas G, Sakai M, et al. Cytosolic Sensors for Pathogenic Viral and Bacterial Nucleic Acids in Fish. Int J Mol Sci (2020) 21(19). doi: 10.3390/ijms21197289

23. Castanier C, Zemirli N, Portier A, Garcin D, Bidere N, Vazquez A, et al. Mavs Ubiquitination by the E3 Ligase TRIM25 and Degradation by the Proteasome is Involved in Type I Interferon Production After Activation of the Antiviral Rig-I-like Receptors. BMC Biol (2012) 10:44. doi: 10.1186/ 1741-7007-10-44

24. Biacchesi S, Merour E, Chevret D, Lamoureux A, Bernard J, Bremont M. Nv Proteins of Fish Novirhabdovirus Recruit Cellular PPM1Bb Protein Phosphatase and Antagonize Rig-I-Mediated IFN Induction. Sci Rep (2017) 7:44025. doi: 10.1038/srep44025

25. Biacchesi S, LeBerre M, Lamoureux A, Louise Y, Lauret E, Boudinot P, et al. Mitochondrial Antiviral Signaling Protein Plays a Major Role in Induction of the Fish Innate Immune Response Against RNA and DNA Viruses. J Virol (2009) 83(16):7815-27. doi: 10.1128/JVI.00404-09

26. Zou J, Chang M, Nie P, Secombes CJ. Origin and Evolution of the RIG-I Like RNA Helicase Gene Family. BMC Evol Biol (2009) 9:85. doi: 10.1186/14712148-9-85

27. Chang M, Collet B, Nie P, Lester K, Campbell S, Secombes CJ, et al. Expression and Functional Characterization of the RIG-I-like Receptors MDA5 and LGP2 in Rainbow Trout (Oncorhynchus Mykiss). J Virol (2011) 85(16):8403-12. doi: 10.1128/JVI.00445-10

28. Gabor KA, Charette JR, Pietraszewski MJ, Wingfield DJ, Shim JS, Millard PJ, et al. A DN-mda5 Transgenic Zebrafish Model Demonstrates That Mda5 Plays an Important Role in Snakehead Rhabdovirus Resistance. Dev Comp Immunol (2015) 51(2):298-304. doi: 10.1016/j.dci.2015.01.006

29. Kawai T, Takahashi K, Sato S, Coban C, Kumar H, Kato H, et al. Ips-1, an Adaptor Triggering RIG-I- and Mda5-mediated Type I Interferon Induction. Nat Immunol (2005) 6(10):981-8. doi: 10.1038/ni1243
30. Meylan E, Curran J, Hofmann K, Moradpour D, Binder M, Bartenschlager $\mathrm{R}$, et al. Cardif is an Adaptor Protein in the RIG-I Antiviral Pathway and is Targeted by Hepatitis C Virus. Nature (2005) 437(7062):1167-72. doi: $10.1038 /$ nature 04193

31. Seth RB, Sun L, Ea CK, Chen ZJ. Identification and Characterization of MAVS, a Mitochondrial Antiviral Signaling Protein That Activates NFkappaB and IRF 3. Cell (2005) 122(5):669-82. doi: 10.1016/j.cell.2005.08.012

32. Xu LG, Wang YY, Han KJ, Li LY, Zhai Z, Shu HB. VISA is an Adapter Protein Required for Virus-Triggered IFN-Beta Signaling. Mol Cell (2005) 19 (6):727-40. doi: 10.1016/j.molcel.2005.08.014

33. Lauksund S, Svingerud T, Bergan V, Robertsen B. Atlantic Salmon Ips-1 Mediates Induction of IFNa1 and Activation of NF-kappaB and Localizes to Mitochondria. Dev Comp Immunol (2009) 33(11):1196-204. doi: 10.1016/ j.dci.2009.06.012

34. Feng H, Liu H, Kong R, Wang L, Wang Y, Hu W, et al. Expression Profiles of Carp Irf-3/-7 Correlate With the Up-Regulation of RIG-I/MAVS/TRAF3/ TBK1, Four Pivotal Molecules in RIG-I Signaling Pathway. Fish Shellfish Immunol (2011) 30(4-5):1159-69. doi: 10.1016/j.fsi.2011.03.002

35. Fitzgerald KA, McWhirter SM, Faia KL, Rowe DC, Latz E, Golenbock DT, et al. Ikkepsilon and TBK1 are Essential Components of the IRF3 Signaling Pathway. Nat Immunol (2003) 4(5):491-6. doi: 10.1038/ni921

36. McWhirter SM, Fitzgerald KA, Rosains J, Rowe DC, Golenbock DT, Maniatis T. Ifn-Regulatory Factor 3-Dependent Gene Expression is Defective in Tbk1-deficient Mouse Embryonic Fibroblasts. Proc Natl Acad Sci USA (2004) 101(1):233-8. doi: 10.1073/pnas.2237236100

37. Sharma S, tenOever BR, Grandvaux N, Zhou GP, Lin R, Hiscott J. Triggering the Interferon Antiviral Response Through an IKK-related Pathway. Science (2003) 300(5622):1148-51. doi: 10.1126/science.1081315

38. Stein C, Caccamo M, Laird G, Leptin M. Conservation and Divergence of Gene Families Encoding Components of Innate Immune Response Systems in Zebrafish. Genome Biol (2007) 8(11):R251. doi: 10.1186/gb-2007-8-11-r251

39. Au WC, Moore PA, Lowther W, Juang YT, Pitha PM. Identification of a Member of the Interferon Regulatory Factor Family That Binds to the Interferon-Stimulated Response Element and Activates Expression of Interferon-Induced Genes. Proc Natl Acad Sci USA (1995) 92(25):1165761. doi: 10.1073/pnas.92.25.11657

40. Holland JW, Bird S, Williamson B, Woudstra C, Mustafa A, Wang T, et al. Molecular Characterization of IRF3 and IRF7 in Rainbow Trout, Oncorhynchus Mykiss: Functional Analysis and Transcriptional Modulation. Mol Immunol (2008) 46(2):269-85. doi: 10.1016/ j.molimm.2008.08.265

41. Petro TM. Ifn Regulatory Factor 3 in Health and Disease. J Immunol (2020) 205(8):1981-9. doi: 10.4049/jimmunol.2000462

42. Sun F, Zhang YB, Liu TK, Gan L, Yu FF, Liu Y, et al. Characterization of Fish IRF3 as an IFN-inducible Protein Reveals Evolving Regulation of IFN Response in Vertebrates. J Immunol (2010) 185(12):7573-82. doi: 10.4049/ jimmunol.1002401

43. Feng H, Zhang QM, Zhang YB, Li Z, Zhang J, Xiong YW, et al. Zebrafish IRF1, IRF3, and IRF7 Differentially Regulate Ifnphi1 and IFNPhi3 Expression Through Assembly of Homo- or Heteroprotein Complexes. J Immunol (2016) 197(5):1893-904. doi: 10.4049/jimmunol.1600159

44. Clark TC, Boudinot P, Collet B. Evolution of the IRF Family in Salmonids. Genes (Basel) (2021) 12(2). doi: 10.3390/genes12020238

45. Nonkwelo C, Ruf IK, Sample J. Interferon-Independent and -Induced Regulation of Epstein-Barr Virus EBNA-1 Gene Transcription in Burkitt Lymphoma. J Virol (1997) 71(9):6887-97. doi: 10.1128/JVI.71.9.68876897.1997

46. Zhang L, Pagano JS. Irf-7, a New Interferon Regulatory Factor Associated With Epstein-Barr Virus Latency. Mol Cell Biol (1997) 17(10):5748-57. doi: $10.1128 / \mathrm{mcb} \cdot 17.10 .5748$

47. Zhang YB, Hu CY, Zhang J, Huang GP, Wei LH, Zhang QY, et al. Molecular Cloning and Characterization of Crucian Carp (Carassius Auratus L.) Interferon Regulatory Factor 7. Fish Shellfish Immunol (2003) 15(5):45366. doi: 10.1016/s1050-4648(03)00025-1

48. Biacchesi S, Merour E, Lamoureux A, Bernard J, Bremont M. Both STING and MAVS Fish Orthologs Contribute to the Induction of Interferon Mediated by RIG-I. PloS One (2012) 7(10):e47737. doi: 10.1371/ journal.pone.0047737 
49. Sun F, Zhang YB, Liu TK, Shi J, Wang B, Gui JF. Fish MITA Serves as a Mediator for Distinct Fish IFN Gene Activation Dependent on IRF3 or IRF7. J Immunol (2011) 187(5):2531-9. doi: 10.4049/jimmunol.1100642

50. Genin P, Vaccaro A, Civas A. The Role of Differential Expression of Human Interferon-a Genes in Antiviral Immunity. Cytokine Growth Factor Rev (2009) 20(4):283-95. doi: 10.1016/j.cytogfr.2009.07.005

51. Altmann SM, Mellon MT, Distel DL, Kim CH. Molecular and Functional Analysis of an Interferon Gene From the Zebrafish, Danio Rerio. J Virol (2003) 77(3):1992-2002. doi: 10.1128/jvi.77.3.1992-2002.2003

52. Boudinot P, Langevin C, Secombes CJ, Levraud JP. The Peculiar Characteristics of Fish Type I Interferons. Viruses (2016) 8(11). doi: $10.3390 / \mathrm{v} 8110298$

53. Ohtani M, Hikima J, Kondo H, Hirono I, Jung TS, Aoki T. Evolutional Conservation of Molecular Structure and Antiviral Function of a Viral Rna Receptor, LGP2, in Japanese Flounder, Paralichthys Olivaceus. J Immunol (2010) 185(12):7507-17. doi: 10.4049/jimmunol.1001850

54. Rao Y, Wan Q, Yang C, Su J. Grass Carp Laboratory of Genetics and Physiology 2 Serves as a Negative Regulator in Retinoic Acid-Inducible Gene I- and Melanoma Differentiation-Associated Gene 5-Mediated Antiviral Signaling in Resting State and Early Stage of Grass Carp Reovirus Infection. Front Immunol (2017) 8:352. doi: 10.3389/fimmu.2017.00352

55. Zhang QM, Zhao X, Li Z, Wu M, Gui JF, Zhang YB. Alternative Splicing Transcripts of Zebrafish Lgp2 Gene Differentially Contribute to IFN Antiviral Response. J Immunol (2018) 200(2):688-703. doi: 10.4049/ jimmunol.1701388

56. Nunez RD, Budt M, Saenger S, Paki K, Arnold U, Sadewasser A, et al. The RNA Helicase DDX6 Associates With RIG-I to Augment Induction of Antiviral Signaling. Int J Mol Sci (2018) 19(7). doi: 10.3390/ijms19071877

57. Lumb JH, Li Q, Popov LM, Ding S, Keith MT, Merrill BD, et al. Ddx6 Represses Aberrant Activation of Interferon-Stimulated Genes. Cell Rep (2017) 20(4):819-31. doi: 10.1016/j.celrep.2017.06.085

58. Oshiumi H, Matsumoto M, Hatakeyama S, Seya T. Riplet/RNF135, a RING Finger Protein, Ubiquitinates RIG-I to Promote Interferon-Beta Induction During the Early Phase of Viral Infection. J Biol Chem (2009) 284(2):807-17. doi: $10.1074 /$ jbc.M804259200

59. Oshiumi H, Miyashita M, Inoue N, Okabe M, Matsumoto M, Seya T. The Ubiquitin Ligase Riplet is Essential for RIG-I-dependent Innate Immune Responses to RNA Virus Infection. Cell Host Microbe (2010) 8(6):496-509. doi: $10.1016 /$ j.chom.2010.11.008

60. Oshiumi H, Miyashita M, Matsumoto M, Seya T. A Distinct Role of Ripletmediated K63-Linked Polyubiquitination of the RIG-I Repressor Domain in Human Antiviral Innate Immune Responses. PloS Pathog (2013) 9(8): e1003533. doi: 10.1371/journal.ppat.1003533

61. Cadena C, Ahmad S, Xavier A, Willemsen J, Park S, Park JW, et al. Ubiquitin-Dependent and -Independent Roles of E3 Ligase RIPLET in Innate Immunity. Cell (2019) 177(5):1187-200 e16. doi: 10.1016/ j.cell.2019.03.017

62. Hayman TJ, Hsu AC, Kolesnik TB, Dagley LF, Willemsen J, Tate MD, et al. RIPLET, and Not TRIM25, is Required for Endogenous RIG-I-Dependent Antiviral Responses. Immunol Cell Biol (2019) 97(9):840-52. doi: 10.1111/ imcb. 12284

63. Lai Y, Liang M, Hu, Zeng Z, Lin H, Yi G, et al. RNF135 is a Positive Regulator of IFN Expression and Involved in RIG-I Signaling Pathway by Targeting Rig-I. Fish Shellfish Immunol (2019) 86:474-9. doi: 10.1016/ j.fsi.2018.11.070

64. Gack MU, Albrecht RA, Urano T, Inn KS, Huang IC, Carnero E, et al. Influenza A Virus NS1 Targets the Ubiquitin Ligase TRIM25 to Evade Recognition by the Host Viral RNA Sensor Rig-I. Cell Host Microbe (2009) 5 (5):439-49. doi: 10.1016/j.chom.2009.04.006

65. Gack MU, Shin YC, Joo CH, Urano T, Liang C, Sun L, et al. Trim25 RINGfinger E3 Ubiquitin Ligase is Essential for RIG-I-mediated Antiviral Activity. Nature (2007) 446(7138):916-20. doi: 10.1038/nature05732

66. Jin $\mathrm{Y}$, Jia $\mathrm{K}$, Zhang $\mathrm{W}$, Xiang $\mathrm{Y}$, Jia P, Liu W, et al. Zebrafish TRIM25 Promotes Innate Immune Response to RGNNV Infection by Targeting 2CARD and RD Regions of RIG-I for K63-Linked Ubiquitination. Front Immunol (2019) 10:2805. doi: 10.3389/fimmu.2019.02805

67. Jahan AS, Biquand E, Munoz-Moreno R, Le Quang A, Mok CK, Wong HH, et al. Otub1 Is a Key Regulator of RIG-I-Dependent Immune Signaling and
Is Targeted for Proteasomal Degradation by Influenza A Ns1. Cell Rep (2020) 30(5):1570-84 e6. doi: 10.1016/j.celrep.2020.01.015

68. Peng Y, Xu R, Zheng X. Hscarg Negatively Regulates the Cellular Antiviral RIG-I Like Receptor Signaling Pathway by Inhibiting Traf3 Ubiquitination Via Recruiting Otub1. PloS Pathog (2014) 10(4):e1004041. doi: 10.1371/ journal.ppat.1004041

69. Xie M, Yin Y, Chen L, Yin A, Liu Y, Liu Y, et al. Scavenger Receptor A Impairs Interferon Response to HBV Infection by Limiting Traf3 Ubiquitination Through Recruiting Otub1. FEBS J (2020) 287(2):310-24. doi: $10.1111 /$ febs. 15035

70. Wies E, Wang MK, Maharaj NP, Chen K, Zhou S, Finberg RW, et al. Dephosphorylation of the RNA Sensors RIG-I and MDA5 by the Phosphatase PP1 is Essential for Innate Immune Signaling. Immunity (2013) 38(3):437-49. doi: 10.1016/j.immuni.2012.11.018

71. Gu M, Zhang T, lin W, Liu Z, Lai R, Xia D, et al. Protein Phosphatase Pp1 Negatively Regulates the Toll-like Receptor- and RIG-I-like ReceptorTriggered Production of Type I Interferon by Inhibiting Irf3 Phosphorylation At Serines 396 and 385 in Macrophage. Cell Signal (2014) 26(12):2930-9. doi: 10.1016/j.cellsig.2014.09.007

72. Kok KH, Lui PY, Ng MH, Siu KL, Au SW, Jin DY. The Double-Stranded RNA-Binding Protein Pact Functions as a Cellular Activator of RIG-I to Facilitate Innate Antiviral Response. Cell Host Microbe (2011) 9(4):299-309. doi: 10.1016/j.chom.2011.03.007

73. Ruan J, Cao Y, Ling T, Li P, Wu S, Peng D, et al. DDX23, an Evolutionary Conserved Dsrna Sensor, Participates in Innate Antiviral Responses by Pairing With TRIF or MAVS. Front Immunol (2019) 10:2202. doi: 10.3389/fimmu.2019.02202

74. Zhang Z, Yuan B, Lu N, Facchinetti V, Liu YJ. Dhx9 Pairs With IPS-1 to Sense Double-Stranded RNA in Myeloid Dendritic Cells. J Immunol (2011) 187(9):4501-8. doi: 10.4049/jimmunol.1101307

75. Poynter SJ, Herrington-Krause S, DeWitte-Orr SJ. Two DExD/H-box Helicases, DDX3 and DHX9, Identified in Rainbow Trout are Able to Bind Dsrna. Fish Shellfish Immunol (2019) 93:1056-66. doi: 10.1016/j.fsi. 2019.07.054

76. Ge R, Zhou Y, Peng R, Wang R, Li M, Zhang Y, et al. Conservation of the STING-Mediated Cytosolic DNA Sensing Pathway in Zebrafish. J Virol (2015) 89(15):7696-706. doi: 10.1128/JVI.01049-15

77. Lu H, Lu N, Weng L, Yuan B, Liu YJ, Zhang Z. Dhx15 Senses DoubleStranded RNA in Myeloid Dendritic Cells. J Immunol (2014) 193(3):136472. doi: 10.4049 /jimmunol.1303322

78. Mosallanejad K, Sekine Y, Ishikura-Kinoshita S, Kumagai K, Nagano T, Matsuzawa A, et al. The DEAH-box Rna Helicase Dhx15 Activates NFKappab and MAPK Signaling Downstream of MAVS During Antiviral Responses. Sci Signal (2014) 7(323):ra40. doi: 10.1126/scisignal.2004841

79. Pattabhi S, Knoll ML, Gale MJr., Loo YM. Dhx15 Is a Coreceptor for RLR Signaling That Promotes Antiviral Defense Against Rna Virus Infection. J Interferon Cytokine Res (2019) 39(6):331-46. doi: 10.1089/jir.2018.0163

80. Liu C, Huang S, Wang X, Wen M, Zheng J, Wang W, et al. The Otubain Yod1 Suppresses Aggregation and Activation of the Signaling Adaptor MAVS Through Lys63-Linked Deubiquitination. J Immunol (2019) 202 (10):2957-70. doi: 10.4049/jimmunol.1800656

81. Vitour D, Dabo S, Ahmadi Pour M, Vilasco M, Vidalain PO, Jacob Y, et al. Polo-Like Kinase 1 (Plk1) Regulates Interferon (Ifn) Induction by MAVS. J Biol Chem (2009) 284(33):21797-809. doi: 10.1074/jbc.M109.018275

82. Zhang L, Liu J, Qian L, Feng Q, Wang X, Yuan Y, et al. Induction of OTUD1 by RNA Viruses Potently Inhibits Innate Immune Responses by Promoting Degradation of the MAVS/TRAF3/TRAF6 Signalosome. PloS Pathog (2018) 14(5):e1007067. doi: 10.1371/journal.ppat.1007067

83. Zhang Z, Wang D, Wang P, Zhao Y, You F. Otud1 Negatively Regulates Type I Ifn Induction by Disrupting Noncanonical Ubiquitination of IRF3. J Immunol (2020) 204(7):1904-18. doi: 10.4049/jimmunol.1900305

84. Ariumi Y. Multiple Functions of DDX3 Rna Helicase in Gene Regulation, Tumorigenesis, and Viral Infection. Front Genet (2014) 5:423. doi: 10.3389/ fgene.2014.00423

85. Oshiumi H, Sakai K, Matsumoto M, Seya T. Dead/H BOX 3 (Ddx3) Helicase Binds the RIG-I Adaptor IPS-1 to Up-Regulate IFN-BetaInducing Potential. Eur J Immunol (2010) 40(4):940-8. doi: 10.1002/eji. 200940203 
86. Soulat D, Burckstummer T, Westermayer S, Goncalves A, Bauch A, Stefanovic A, et al. The DEAD-box Helicase DDX3X is a Critical Component of the TANK-binding Kinase 1-Dependent Innate Immune Response. EMBO J (2008) 27(15):2135-46. doi: 10.1038/emboj.2008.126

87. Loureiro M, Zorzetto-Fernandes AL, Radoshitzky S, Chi X, Dallari S, Marooki N, et al. Ddx3 Suppresses Type I Interferons and Favors Viral Replication During Arenavirus Infection. PloS Pathog (2018) 14(7). doi: 10.1371/journal.ppat.1007125. doi: ARTN e1007125.

88. Liu J, Huang X, Yu Y, Zhang J, Ni S, Hu Y, et al. Fish DDX3X Exerts Antiviral Function Against Grouper Nervous Necrosis Virus Infection. Fish Shellfish Immunol (2017) 71:95-104. doi: 10.1016/j.fsi.2017.09.068

89. Merour E, Jami R, Lamoureux A, Bernard J, Bremont M, Biacchesi S. A20 (tnfaip3) is a Negative Feedback Regulator of RIG-I-Mediated Ifn Induction in Teleost. Fish Shellfish Immunol (2019) 84:857-64. doi: 10.1016/ j.fsi.2018.10.082

90. Lin R, Yang L, Nakhaei P, Sun Q, Sharif-Askari E, Julkunen I, et al. Negative Regulation of the Retinoic Acid-Inducible Gene I-Induced Antiviral State by the Ubiquitin-Editing Protein A20. J Biol Chem (2006) 281(4):2095-103. doi: $10.1074 / j b c . M 510326200$

91. Saitoh T, Yamamoto M, Miyagishi M, Taira K, Nakanishi M, Fujita T, et al. A20 is a Negative Regulator of IFN Regulatory Factor 3 Signaling. J Immunol (2005) 174(3):1507-12. doi: 10.4049/jimmunol.174.3.1507

92. Zhu C, Xiao F, Hong J, Wang K, Liu X, Cai D, et al. Eftud2 Is a Novel Innate Immune Regulator Restricting Hepatitis C Virus Infection Through the RIG-I/MDA5 Pathway. J Virol (2015) 89(13):6608-18. doi: 10.1128/ JVI.00364-15

93. Li Z, Liu G, Sun L, Teng Y, Guo X, Jia J, et al. Ppm1a Regulates Antiviral Signaling by Antagonizing TBK1-Mediated STING Phosphorylation and Aggregation. PloS Pathog (2015) 11(3):e1004783. doi: 10.1371/ journal.ppat.1004783

94. Xiang W, Zhang Q, Lin X, Wu S, Zhou Y, Meng F, et al. Ppmla Silences Cytosolic RNA Sensing and Antiviral Defense Through Direct Dephosphorylation of MAVS and TBK1. Sci Adv (2016) 2(7):e1501889. doi: 10.1126/sciadv.1501889

95. Zhao Y, Liang L, Fan Y, Sun S, An L, Shi Z, et al. Ppm1b Negatively Regulates Antiviral Response Via Dephosphorylating Tbk1. Cell Signal (2012) 24 (11):2197-204. doi: 10.1016/j.cellsig.2012.06.017

96. Zhang K, Zhang Y, Xue J, Meng Q, Liu H, Bi C, et al. Ddx19 Inhibits Type I Interferon Production by Disrupting Tbk1-Ikkepsilon-Irf3 Interactions and Promoting TBK1 and IKKepsilon Degradation. Cell Rep (2019) 26(5):125872 e4. doi: 10.1016/j.celrep.2019.01.029

97. Mankouri J, Fragkoudis R, Richards KH, Wetherill LF, Harris M, Kohl A, et al. Optineurin Negatively Regulates the Induction of IFNbeta in Response to RNA Virus Infection. PloS Pathog (2010) 6(2):e1000778. doi: 10.1371/ journal.ppat.1000778

98. Gleason CE, Ordureau A, Gourlay R, Arthur JS, Cohen P. Polyubiquitin Binding to Optineurin is Required for Optimal Activation of TANK-binding Kinase 1 and Production of Interferon Beta. J Biol Chem (2011) 286 (41):35663-74. doi: 10.1074/jbc.M111.267567

99. Genin P, Cuvelier F, Lambin S, Corte-Real Filipe J, Autrusseau E, Laurent C, et al. Optineurin Regulates the Interferon Response in a Cell CycleDependent Manner. PloS Pathog (2015) 11(4):e1004877. doi: 10.1371/ journal.ppat.1004877

100. Li Y, Jiang N, Mao Y, Zhang W, Xiao J, Wu X, et al. Chicken Optineurin Suppresses MDA5-mediated Interferon Beta Production. Poult Sci (2021) 100(1):9-18. doi: 10.1016/j.psj.2020.08.020

101. Outlioua A, Pourcelot M, Arnoult D. The Role of Optineurin in Antiviral Type I Interferon Production. Front Immunol (2018) 9:853. doi: 10.3389/ fimmu.2018.00853

102. Lu LF, Zhou XY, Zhang C, Li ZC, Chen DD, Liu SB, et al. Zebrafish RPZ5 Degrades Phosphorylated Irf7 To Repress Interferon Production. J Virol (2019) 93(21). doi: 10.1128/JVI.01272-19

103. Cai Z, Zhang MX, Tang Z, Zhang Q, Ye J, Xiong TC, et al. Usp22 Promotes Irf3 Nuclear Translocation and Antiviral Responses by Deubiquitinating the Importin Protein Kpna2. J Exp Med (2020) 217(5). doi: 10.1084/ jem.20191174

104. Liu Q, Wu Y, Qin Y, Hu J, Xie W, Qin FX, et al. Broad and Diverse Mechanisms Used by Deubiquitinase Family Members in Regulating the
Type I Interferon Signaling Pathway During Antiviral Responses. Sci Adv (2018) 4(5):eaar2824. doi: 10.1126/sciadv.aar2824

105. Barber MR, Aldridge JR Jr, Webster RG, Magor KE. Association of RIG-I With Innate Immunity of Ducks to Influenza. Proc Natl Acad Sci USA (2010) 107(13):5913-8. doi: 10.1073/pnas.1001755107

106. Xu L, Yu D, Fan Y, Peng L, Wu Y, Yao YG. Loss of RIG-I Leads to a Functional Replacement With MDA5 in the Chinese Tree Shrew. Proc Natl Acad Sci USA (2016) 113(39):10950-5. doi: 10.1073/pnas.1604939113

107. Streicher F, Jouvenet N. Stimulation of Innate Immunity by Host and Viral Rnas. Trends Immunol (2019) 40(12):1134-48. doi: 10.1016/ j.it.2019.10.009

108. Chang MX, Zhang J. Alternative Pre-mRNA Splicing in Mammals and Teleost Fish: A Effective Strategy for the Regulation of Immune Responses Against Pathogen Infection. Int J Mol Sci (2017) 18(7). doi: 10.3390/ ijms 18071530

109. Zhou Y, He C, Wang L, Ge B. Post-Translational Regulation of Antiviral Innate Signaling. Eur J Immunol (2017) 47(9):1414-26. doi: 10.1002/ eji.201746959

110. Esser-Nobis K, Hatfield LD, Gale MJr. Spatiotemporal Dynamics of Innate Immune Signaling Via RIG-I-like Receptors. Proc Natl Acad Sci USA (2020) 117(27):15778-88. doi: 10.1073/pnas.1921861117

111. Pourcelot M, Arnoult D. Mitochondrial Dynamics and the Innate Antiviral Immune Response. FEBS J (2014) 281(17):3791-802. doi: 10.1111/febs.12940

112. Yoshinaka T, Kosako H, Yoshizumi T, Furukawa R, Hirano Y, Kuge O, et al. Structural Basis of Mitochondrial Scaffolds by Prohibitin Complexes: Insight Into a Role of the Coiled-Coil Region. iScience (2019) 19:1065-78. doi: 10.1016/j.isci.2019.08.056

113. Chang MX. The Negative Regulation of Retinoic Acid-Inducible Gene I (RigI)-Like Receptors (Rlrs) Signaling Pathway in Fish. Dev Comp Immunol (2021) 119:104038. doi: 10.1016/j.dci.2021.104038

114. Li W, Cao Y, Chen Z, Tan Y, Dai Y, Wei J, et al. Black Carp Tradd Suppresses Mavs/Ifn Signaling During the Innate Immune Activation. Fish Shellfish Immunol (2021) 111:83-93. doi: 10.1016/j.fsi.2021.01.006

115. Winton J, Batts W, deKinkelin P, LeBerre M, Bremont M, Fijan N. Current Lineages of the Epithelioma Papulosum Cyprini (Epc) Cell Line are Contaminated With Fathead Minnow, Pimephales Promelas, Cells. J Fish Dis (2010) 33(8):701-4. doi: 10.1111/j.1365-2761.2010.01165.x

116. Jorgensen LVG. Zebrafish as a Model for Fish Diseases in Aquaculture. Pathogens (2020) 9(8). doi: 10.3390/pathogens9080609

117. Levraud JP, Palha N, Langevin C, Boudinot P. Through the Looking Glass: Witnessing Host-Virus Interplay in Zebrafish. Trends Microbiol (2014) 22 (9):490-7. doi: 10.1016/j.tim.2014.04.014

118. Varela M, Figueras A, Novoa B. Modelling Viral Infections Using Zebrafish: Innate Immune Response and Antiviral Research. Antiviral Res (2017) 139:59-68. doi: 10.1016/j.antiviral.2016.12.013

119. Onomoto K, Onoguchi K, Yoneyama M. Regulation of RIG-I-like ReceptorMediated Signaling: Interaction Between Host and Viral Factors. Cell Mol Immunol (2021) 18(3):539-55. doi: 10.1038/s41423-020-00602-7

120. Liu SQ, Cai X, Wu JX, Cong Q, Chen X, Li T, et al. Phosphorylation of Innate Immune Adaptor Proteins MAVS, STING, and TRIF Induces IRF3 Activation. Science (2015) 347(6227):1217-U17. doi: 10.1126/science. aaa2630

121. Zheng N, Shabek N. Ubiquitin Ligases: Structure, Function, and Regulation. Annu Rev Biochem (2017) 86:129-57. doi: 10.1146/annurev-biochem060815-014922

122. Miyamoto M, Komuro A. PACT is Required for MDA5-mediated Immunoresponses Triggered by Cardiovirus Infection Via Interaction With Lgp2. Biochem Biophys Res Commun (2017) 494(1-2):227-33. doi: 10.1016/j.bbrc.2017.10.048

123. Vaughn LS, Chukwurah E, Patel RC. Opposite Actions of Two DsrnaBinding Proteins PACT and TRBP on RIG-I Mediated Signaling. Biochem J (2021) 478(3):493-510. doi: 10.1042/BCJ20200987

124. Taschuk F, Cherry S. Dead-Box Helicases: Sensors, Regulators, and Effectors for Antiviral Defense. Viruses (2020) 12(2). doi: 10.3390/v12020181

125. O’Loughlin T, Kruppa AJ, Ribeiro ALR, Edgar JR, Ghannam A, Smith AM, et al. Optn Recruitment to a Golgi-proximal Compartment Regulates Immune Signalling and Cytokine Secretion. J Cell Sci (2020) 133(12). doi: $10.1242 /$ jcs. 239822 
126. Dehler CE, Lester K, Della Pelle G, Jouneau L, Houel A, Collins C, et al. Viral Resistance and IFN Signaling in STAT2 Knockout Fish Cells. J Immunol (2019) 203(2):465-75. doi: 10.4049/jimmunol.1801376

127. Kim MS, Shin MJ, Kim KH. Increase of Viral Hemorrhagic Septicemia Virus Growth by Knockout of IRF9 Gene in Epithelioma Papulosum Cyprini Cells. Fish Shellfish Immunol (2018) 83:443-8. doi: 10.1016/j.fsi. 2018.09.025

128. Sifuentes-Romero I, Milton SL, Garcia-Gasca A. Post-Transcriptional Gene Silencing by RNA Interference in non-Mammalian Vertebrate Systems: Where do We Stand? Mutat Res (2011) 728(3):158-71. doi: 10.1016/ j.mrrev.2011.09.001

129. Garcia-Moreno D, Tyrkalska SD, Valera-Perez A, Gomez-Abenza E, PerezOliva AB, Mulero V. The Zebrafish: A Research Model to Understand the Evolution of Vertebrate Immunity. Fish Shellfish Immunol (2019) 90:215-22. doi: 10.1016/j.fsi.2019.04.067
130. Vila IK, Fretaud M, Vlachakis D, Laguette N, Langevin C. Animal Models for the Study of Nucleic Acid Immunity: Novel Tools and New Perspectives. J Mol Biol (2020) 432(20):5529-43. doi: 10.1016/j.jmb.2020.08.016

Conflict of Interest: The authors declare that the research was conducted in the absence of any commercial or financial relationships that could be construed as a potential conflict of interest.

Copyright (c) 2021 Jami, Mérour, Lamoureux, Bernard, Millet and Biacchesi. This is an open-access article distributed under the terms of the Creative Commons Attribution License (CC BY). The use, distribution or reproduction in other forums is permitted, provided the original author(s) and the copyright owner(s) are credited and that the original publication in this journal is cited, in accordance with accepted academic practice. No use, distribution or reproduction is permitted which does not comply with these terms. 